\title{
Identité post mortem et nouvelles pratiques mémoriales en ligne
}

L'identité du créateur de la page mémoriale sur Facebook

\author{
Fanny GEORGES
}

\section{Introduction}

Avec le développement des réseaux socionumériques (RSN), le web est devenu un cadre de présentation ordinaire des individus. Or, le vieillissement des usagers du web et la disparition des personnes ayant créé des pages de profil soulèvent la question des données post mortem (Merzeau, 2009). Que deviennent les données laissées sur Internet par les individus après leur mort? Les défunts ont-ils aussi une identité numérique ? En quoi ce phénomène est-il lié à celui de leur identité numérique de leur vivant ? La permanence matérielle des données numériques ne dépendant pas de la présence de l'individu, mais du serveur et du service web propriétaire, l'identité numérique de l'usager peut rester identique à ce qu'elle était de son vivant si personne n'intervient. Or, la présence de ces traces sur internet peut incommoder les proches en leur rappelant constamment le décès de leur défunt. De surcroît, les services du web participatif comme Facebook relancent d'autant plus les contacts d'un sujet que ce dernier est inactif. Ne pouvant différencier un usager défunt d'un usager simplement inactif sur son compte, Facebook envoie des notifications automatiques de reprise de contact aux proches des défunts, provoquant un sentiment d'étrangeté et avivant la douleur du deuil. Les pages web peuvent également être créées à l'initiative des proches, pour l'annonce du décès et des funérailles (Wrona, 2011), ou encore pour exprimer leur douleur à destination des vivants comme du défunt. Ces questions interrogent les limites de l'identité numérique, après le décès des individus. Si l'étude de ce domaine de l'identité numérique est bien représentée dans la recherche actuelle, la question du devenir de ces identités et de leur transformation après le décès des usagers est encore émergeant. Il est voué à prendre de plus en plus d'importance dans la société du fait du vieillissement des usagers du web. 
F. GEORGES — Identité post mortem - 52

Cette contribution présente les premières étapes d'une recherche en cours sur les profils mémoriaux, qui s'appuie sur une approche sémiopragmatique (Meunier et Peraya, 2004) ainsi que sur des cadres d'analyse de l'identité numérique. Dans l'objectif de mieux comprendre, à long terme, la gestion des traces des défunts par leur entourage, cette première approche propose une analyse des représentations du créateur de la page mémoriale dans Facebook.

Cet article propose de mettre en perspective une approche sémiopragmatique de l'identité numérique (cf. première section) avec une approche typologique des sites de présentation des défunts prenant pour critère discriminant la relation subjective entre le sujet (créateur de la page de profil) et l'objet de la page (le défunt) (cf. deuxième section). Le rapprochement de ces deux domaines permet de mettre en évidence, dans une troisième section, les enjeux de l'identité du créateur de la page mémoriale.

\section{L'identité numérique interrogée sous l'angle sémiopragmatique}

L'«identité numérique» constitue un phénomène sociotechnique apparu avec la communication numérique et les premières formes de profils d'internautes sur le web (Georges, 2009a). Les pages qui présentent l'identité des usagers interrogent à la fois le transfert des modalités de présentation de soi sur le web (Georges, 2009; Coutant et Stenger, 2010) et les problématiques de gestion des données de l'usager et de privacy, ou propriété des données personnelles, et de droit à l'oubli (Ertzcheid et al. 2013).

Partant d'une approche sémiopragmatique, nous avons interrogé l'identité numérique du vivant des usagers à travers un vaste corpus : pages de profils utilisateurs dans les forums, pages personnelles, jeux vidéo, blogs et pages de profil du web participatif. Nous avons ainsi défini l'identité numérique comme l'ensemble des signes qui manifestent l'utilisateur à l'écran. Le relevé et l'analyse de ces informations permettent de proposer différentes classifications répondant à des critères discriminants.

En s'appuyant sur l'approche sémiotique pragmatique de Charles $\mathrm{S}$. Peirce, on peut penser la représentation numérique de l'identité comme un «schéma-silhouette de soi» (Peirce, 1978: 120-121)

GEORGES, Fanny (2014) «Identité post mortem et nouvelles pratiques memoriales en ligne. L'identité du créateur de la page memoriale. », Les Cahiers du Gerse, Presses de l'Université du Québec. 
donné par l'interprète sous la forme d'une production discursive multimodale (le representamen de C.-S. Peirce). La triade icône-indicesymbole peircienne est particulièrement bien adaptée à l'étude de ces productions multimodales ayant pour objet l'image de soi.

La relation symbolique est inséparable du processus de réception et d'interprétation: dans le cadre du numérique, les usagers coconstruisent dans les deux cas des schèmes interprétatifs, sous forme de conventions établies entre des usagers d'un petit groupe (ex. : l'usager règle par défaut son statut à «indisponible » dans ICQ, ses contacts proches savent que cela ne signifie pas qu'il est indisponible pour discuter, tandis que ses contacts plus éloignés prendront cette notification en son sens initial), ou procédant d'usages convenus plus généralement (ex. s'exprimer en lettres capitales équivaut à crier).

L'étude de la relation iconique permet de mettre en évidence la relation d'analogie entre le profil numérique de l'usager et l'image de soi en pensée. Ainsi, le modèle de la métaphore du profil (Georges, 2010) interroge la façon avec laquelle le site web donne forme à l'image de soi à travers les représentations numériques de l'identité ainsi définies. Cette approche propose consécutivement une lecture de la manière dont le profil de l'usager peut influencer l'image que se fait le sujet de sa propre identité personnelle. Le profil de l'usager est ainsi interprété comme un miroir dans lequel le sujet voit une image de lui-même qui se modifie malgré lui (que ce soit de son fait ou de celui d'un tiers) et qu'il peut réajuster en fonction de l'image qu'il souhaite donner.

La notion d'indice offre quant à elle la possibilité de distinguer les signes qui sont une trace de l'activité du sujet, de ses amis ou du site web, permettant d'interroger le lien de contiguiité entre le sujet et sa page de profil. Le modèle de l'identité numérique a ainsi permis de discerner les signes saisis directement par l'usager pour créer sa présentation de soi numérique (nom, prénom, genre, photographies, centres d'intérêt, activités, humeur etc.) de ceux qui sont le produit d'une captation et d'une notification du site web sur la page de profil de l'usager - que son contenu soit textuel (identité agissante : $\mathrm{x}$ est désormais ami avec $\mathrm{y}, \mathrm{x}$ a téléchargé telle application etc.) et/ou quantifié (identité calculée: nombre d'amis, nombre de groupes, nombre de «j'aime»). Une approche quantifiée de ce modèle a 
F. GEORGES — Identité post mortem - 54

montré que le web participatif se caractérise par une perte d'emprise de l'utilisateur sur sa représentation, en raison de la participation des «amis » ou «contacts» et du système informatique au processus d'énonciation (Georges, 2009) : même si l'utilisateur ne saisit aucune information (la condition préalable étant qu'il ait créé un profil), le système informatique du site web délivre des informations sur son identité en continu.

Cette triple lecture à la lumière de la triade indice-icône-symbole permet donc de mettre en évidence trois dimensions des productions discursives ayant pour objet soi-même : 1) la dimension coconstruite des usages, aboutissant à des normes (qu'elles soient locales ou générales), dans le double mouvement d'interprétation et de production de l'interprète-sujet de la représentation numérique de son identité ; 2) la participation des pages de profil à la construction de l'image de soi en pensée; 3) la perte d'emprise croissante de l'usager sur sa représentation, au profit des «contacts » de l'usager et du système informatique. Des travaux ont montré que les informations que le sujet déclare lui-même pour se présenter sont jugées moins «authentiques» par les «amis» de l'usager que celles qui sont saisies par les tiers; par un processus interprétatif iconique surprenant, le caractère esthétique des photos de profil des «amis» qui publient sur le mur d'un usager de Facebook auraient un rôle décisif sur l'image de sympathie de ce dernier (Walther et. Al. 2007). Quelle que soit la volonté de l'utilisateur, l'identité numérique continue de se construire, par une forme de délégation de la présentation de soi au dispositif technique et à la communauté de ses «amis ».

\section{Typologie des sites de présentation des défunts}

L'étude du contexte post mortem de l'identité numérique permet d'interroger les limites des résultats que je viens de présenter en ce qui concerne l'étude de l'identité numérique du vivant de l'usager. Afin de mettre en évidence la relation d'énonciation, une première approche a consisté à distinguer trois catégories de sites présentant des données identitaires sur les défunts sur internet (Georges et Julliard, 2014). Cette première analyse s'est appuyée sur le modèle 
relationnel de la mort ${ }^{1}$ élaboré par Vladimir Jankélévitch tel qu'appliqué par Alain Rabatel et Marie-Laure Florea concernant les modalités d'annonce de la mort au sein des médias. Cette distinction permet aux chercheurs de montrer que, si les médias traditionnels se focalisent sur la «mort en troisième personne», les nouvelles technologies offrent des voies d'expression inédites à la mort «en deuxième » et «première personne[s]» (Jankélévitch, 1977), c'est-à-dire la mort du proche, d'une part, et la mort propre vécue au futur, d'autre part (Rabatel et Florea, 2011). Nous nous sommes également appuyées sur les catégorisations de Sofka (2009) et Haverinen (2010) qui prennent respectivement pour critère discriminant le caractère intentionnel de la visée mémoriale des profils (les usagers ont-ils voulu ou non que leur profil perdure après leur mort?), et le caractère dédié de la plateforme (est-elle ou non dédiée aux usages mémoriaux?). Interrogeant l'identité numérique post mortem sous l'angle de la relation du sujet à l'objet de l'énonciation, nous avons donc distingué :

1. les hommages créés par des proches postérieurement au décès ( $3^{\mathrm{e}}$ personne),

2. les profils créés par les usagers de leur vivant, et transformés par les proches postérieurement au décès de l'usager $\left(2^{\mathrm{e}}\right.$ personne) en lieu de recueil et d'hommage,

3. les sites qui proposent à l'usager de créer et gérer lui-même ses données de son vivant dans la perspective de son décès à venir ( $1^{\text {re }}$ personne).

La première catégorie des «hommages créés par des proches postérieurement au décès » est la plus ancienne. Dès les années 1990, on observe en effet l'existence de cyber-cimetières sur Internet. Construits dans un univers graphique inspiré des cimetières traditionnels, ils permettent aux proches de créer des mémoriaux en ligne dans le but de rendre hommage aux défunts (de Vries et Rutherdorf, 2004). Certains sont spécialisés, comme les cimetières dédiés aux morts du SIDA, aux célébrités (Hall et Reid, 2009), aux

${ }^{1}$ V. Jankélévitch distingue la mort en troisième personne (« la mort en général, la mort abstraite et anonyme »), la mort en deuxième personne (la mort d'un proche, la mort d'un être cher) et la mort en première personne (ma mort singulière, pour laquelle personne ne peut me remplacer) (Jankélévitch, $1977: 25)$ 
F. GEORGES — Identité post mortem - 56

victimes de guerre (Walter et al., 2012) ou encore aux animaux domestiques (Blando et al., 2004). Ces mémoriaux permettent de choisir des «tombes », de les «fleurir », de « brûler » de l'encens et de rédiger des hommages (Bell, 2006). Ils sont le lieu de la relation de la mort «à la $3^{e}$ personne» pour réinvestir, dans le contexte du numérique, le modèle des trois relations à la mort définies par le philosophe V. Jankélévitch.

La deuxième catégorie réfère à la mort à la deuxième personne au sein des réseaux socionumériques (Facebook, Myspace) en particulier. Bien que ces sites ne soient pas initialement dédiés à la mémoire des défunts, la mort y est toutefois présente à travers les profils des usagers décédés. Facebook, par les notifications impromptues d'invitation à reprendre contact avec des amis décédés, provoque une modification du processus de deuil (Wrona, 2011) en avivant par ces relances la douleur des usagers (Pène, 2011). Si le site a mis en place une fonctionnalité permettant, sous réserve de fournir une preuve officielle de décès, de supprimer ou transformer la page en mémorial statique dépourvu de relances, la fonctionnalité est peu connue donc peu utilisée (Odom, 2011). Selon S. Pène, Facebook est le lieu de trois formes d'annonces de la mort: le faire-part, les pages de profil de personnes décédées, et les commémorations de catastrophes, de crimes, d'accidents et de maladies (Pène, 2011). Ces trois formes d'annonce de la mort présentent différents enjeux affectifs et symboliques, notamment en raison de leur nouveauté en regard des pages des cimetières virtuels: elles peuvent présenter des signes indiciels du défunt (signes d'activité, humeurs, photos téléchargées, notifications d'usage d'applications, publications sur le mur), et en cela se rapproche de la mort à la $1^{\text {re }}$ personne.

La troisième catégorie rassemble les sites qui proposent directement à l'utilisateur de créer son mémorial de son vivant. La tentative de communiquer avec ses proches après son décès apparait assez tôt dans les usages, dès le début des années 1990, avec des détournements de services d'envoi de courriels programmés. Un message à destination d'un ou plusieurs proches est rédigé par l'usager qui prévoit son envoi pour une date ultérieure, en l'occurrence après la date supposée de son décès. Dédiés à la présentation post mortem de soi-même par soi-même, des sites internet de plus en plus sophistiqués se sont développés ces dernières années

GEORGES, Fanny (2014) «Identité post mortem et nouvelles pratiques memoriales en ligne. L'identité du créateur de la page memoriale. », Les Cahiers du Gerse, Presses de l’Université du Québec. 
(Lachut, 2009; Foong et Kera 2008). Dans cette catégorie, les représentations du défunt sont entièrement choisies par les défunts avant leur décès.

Ainsi, cette classification montre que les nouvelles technologies sont bien le lieu de l'expression de la mort à la $1^{\text {re }}$ et à la $2^{\mathrm{e}}$ personne, même si la mort à la $3^{3}$ personne n'est pas exclue. En s'appuyant sur les travaux qui montrent la perte d'emprise de l'usager sur la représentation de soi, on peut en effet souligner la tendance du web participatif à valoriser une relation de la mort à la $3^{e}$ personne. Ces différents contextes d'énonciation invitent à s'interroger sur le sujet énonciateur et producteur de la page d'hommage au défunt. Les approches de l'identité numérique précédemment exposées vont permettre de problématiser cet aspect du phénomène.

\section{L'identité du producteur de la page mémoriale}

La typologie présentée dans la section précédente a permis de mettre en évidence la question de l'énonciation dans les pages mémoriales. Dans les réseaux socionumériques en particulier (catégorie 2), on observe une rencontre entre les représentations du défunt à la deuxième et à la première personne. En particulier, les pages Facebook présentant des hommages à des personnes défuntes, qu'elles soient créées par l'usager de son vivant ou par son entourage après son décès, posent la question de la rencontre entre le sujet et l'objet de la représentation. Nous allons repositionner ce phénomène dans le contexte de l'étude de l'identité numérique, afin d'expliciter la problématique de l'énonciation.

En premier lieu, si la dimension iconique de l'image de soi ne peut plus avoir d'influence sur la construction de l'image de soi après la mort, en revanche elle en a une sur l'image que les autres ont de soi. Dans le cas des mémoriaux créés ex nibilo par les proches postérieurement au décès de l'usager (cf. deuxième catégorie de la typologie), des sites comme Facebook - non spécialisés sur les pages mémoriales - impliquent que les créateurs des pages mémoriales se présentent dans une grande proximité à l'objet (le défunt) de la page en question. Par exemple, la page mémoriale « Repose en paix (Nom Prénom)» est créée par un utilisateur «Repose en paix (Nom Prénom) », qui n'est pas la personne objet de la page (le défunt Nom Prénom), mais bien un ou plusieurs proches. Toutefois, l'inclusion 
F. GEORGES — Identité post mortem — 58

des nom et prénom dans le nom du groupe présente une certaine ambigüité porteuse de signification. Qui plus est, chaque action de cet utilisateur-créateur s'opère sous le nom du groupe. Ainsi verra-ton «Repose en paix (Nom Prénom)» publier des commentaires et des photographies ou encore «liker» des publications sur la page d'hommage.

En second lieu, l'identité post mortem peut être envisagée comme s'inscrivant dans la continuité du processus de délégation de la présentation de soi au système informatique et aux «contacts». Que se passe-t-il lorsque l'usager décède, et ne peut plus être sujet de la production discursive de sa page? Son identité numérique continue-telle de se construire? Jed Brubaker et Janet Vertesi (Brubaker et Vertesi, 2010) ont montré que oui : après le décès d'un usager, son identité numérique continue de se construire par l'action des proches qui entretiennent le souvenir du défunt. Leurs travaux montrent même que ce processus engendre un phénomène spécifique de «récalcitrance » selon lequel les proches témoignent du sentiment que le défunt, à travers sa page de profil Facebook, reste «persistant et actif ». L'usage de profils mémoriaux peut avoir des conséquences sur le vécu même du deuil, car il entretient la détresse tout en atténuant la douleur et prolonge le processus de deuil (Brubaker et Hayes, 2011).

Le phénomène de la « récalcitrance des données identitaires » relevé par Brubaker et Vertesi (2010) peut être en partie expliqué par l'importance croissante prise par les dimensions agissantes et calculées de l'identité numérique. Toutefois, ceci ne peut être valable qu'en ce qui concerne les pages de profils créées de leur vivant par les usagers. Puisque les modalités d'énonciation changent dans les mémoriaux créés ex nibilo par les proches postérieurement au décès de l'usager (cf. deuxième catégorie de la typologie), on peut se demander comment cette énonciation ambiguë se noue entre, d'une part, les proches, créateurs de la page et sujet de l'énonciation, et d'autre part, le défunt-objet de l'énonciation, contenu matériellement dans le sujet de l'énonciation.

Pour mettre en œuvre cette première analyse des enjeux du dispositif énonciatif sur les formes de l'hommage post mortem, nous avons réuni 15 pages mémoriales issues du réseau socionumérique Facebook. Ces 15 pages mémoriales font l'objet d'une interrogation portant sur le

GEORGES, Fanny (2014) «Identité post mortem et nouvelles pratiques memoriales en ligne. L'identité du créateur de la page memoriale. », Les Cahiers du Gerse, Presses de l'Université du Québec. 
sujet producteur de la page. Dans cette première approche, nous n'avons pas eu recours à un traitement automatique des données pour constituer le corpus, ni pour l'analyser. Les pages de profils des défunts ont été recueillies par une recherche manuelle, par le biais du moteur de recherche de Facebook, à l'aide des mots clés «Hommage » et «Repose en paix». Par cette méthode, nous avons obtenu de nombreux résultats, dont nous avons utilisé les premières occurrences. Celles-ci présentent des profils mémoriaux créés par les proches, fans ou inconnus, en hommage à un ou plusieurs défunts. Sur ces 15 pages, 12 sont publiques, 2 à accès restreint, 1 semipublique. Le caractère public des pages atténue le caractère dit «sensible» de ces données, mais elles sont toutefois anonymisées dans cet article. Pour les pages à accès restreint ou semi-publiques, nous n'avons pas analysé les données paramétrées comme confidentielles.

Pour analyser l'énonciation opérée par le créateur de la page, nous avons recueilli les données suivantes :

- le titre de la page,

- la catégorie de la page (groupe, personnalité, profil personnel),

- les publications produites par le créateur de la page, c'est-à-dire ayant pour producteur le « ligateur autonyme »'.

Plus spécifiquement, dans ce dernier ensemble, nous avons recueilli les informations sur le sujet de l'énonciation dans les textes publiés ( je», "nous», formule passive, signature). Sur les 15 pages analysées, 10 présentent un sujet producteur différent de l'objet de la page, les 5 autres étant des pages de profil ayant pour objet l'usager de la page, et présentant un hommage à un tiers défunt.

En complément, sept entretiens exploratoires portant sur la page rendant compte du décès d'un proche (ami ou personne de la famille) ont été conduits auprès de jeunes adultes.

\footnotetext{
${ }^{2}$ Le « ligateur autonyme » désigne l'ensemble formé par la photo de profil ainsi que le titre de la page qui est aussi l'identifiant utilisateur.
} 
F. GEORGES — Identité post mortem — 510

\subsection{Enjeux de la catégorie de page choisie dans le positionnement énonciatif du créateur}

La catégorie de page utilisée dans Facebook pour créer le mémorial implique une certaine relation entre le créateur de la page et l'objet de la page. Sur les 15 pages analysées, 7 sont des pages « groupe », 6 sont des profils personnels, et deux des profils professionnels (actor/producer et teacher).

Parmi les 6 pages « groupe », 5 sont publiques, 1 semi-privée (seule la liste des membres est visible). Leur intitulé présente clairement l'objet de la page mémoriale - ceci étant dû également aux mots-clés de notre recherche. Le sujet opérateur de l'énonciation ne peut évidemment coïncider dans les faits avec l'objet de la page (le défunt). Toutefois, cette différence qui n'est pas problématique ailleurs (par exemple, dans les cimetières numériques de la première catégorie de notre typologie), le devient dans le cas des pages de cette catégorie dans Facebook. N'étant pas conçues pour porter sur une identité qui n'est pas celle du sujet producteur (dans les pages groupes ou communautés, le créateur de la page fait partie du groupe), ces pages doivent faire l'objet d'un détournement - d'une appropriation sémiotique (Bonenfant, ) - pour que le fait de publier sous le nom du groupe ne présente pas de confusion avec l'objet du groupe (le défunt). Dans le cadre de cette stratégie, les pages rassemblent des procédés utilisant les expressions «Hommage à » ou «Repose en paix », complétées dans 3 cas de figure par des formules ( $\mathrm{A}$ jamais dans nos cœurs») ou par des expressions affectueuses ("mon amour », « notre plus bel ange »).

Deux pages du corpus relèvent de la catégorie «actor/producer» et «teacher». L'une utilise l'expression « Hommage à », l'autre « Repose en paix ». Ces pages sont dédiées à la présentation d'une personnalité célèbre ou reconnue, et permet l'hommage à la troisième personne. $\mathrm{Si}$ on ne distingue pas formellement l'identité du créateur de la page de profil de la célébrité en question, cette différence semble en revanche implicite dans les usages, et n'est pas sans être sujette à questionnement de la part des participants, comme nous le montrerons plus loin.

Sur les pages de profils personnels présentant une formule dédiée à l'hommage funèbre, on distingue deux ensembles : sur les 6 profils de

GEORGES, Fanny (2014) «Identité post mortem et nouvelles pratiques memoriales en ligne. L'identité du créateur de la page memoriale. », Les Cahiers du Gerse, Presses de l’Université du Québec. 
cette catégorie, 3 mentionnent, entre parenthèse et à côté du nom et prénom de l'usager, l'expression « Repose en paix » suivie du prénom ou du prénom nom du défunt. Les 3 autres présentent la formule «Repose en paix » entre parenthèses après les nom et prénom du défunt.

Le premier ensemble, qui présente un procédé semblant usuel au vu du grand nombre d'occurrences dans cet échantillon réduit, réfère à un hommage à un défunt temporaire sur le profil d'une personne de son entourage. Dans l'échantillon que nous avons consulté, en un mois d'intervalle, l'un des 3 profils présentant cette mention l'a supprimée.

La seule page de notre corpus présentant un profil d'usager défunt ne fait mention ni de la date du décès, et ne présente aucune publication textuelle: seules des photographies de la défunte sont présentées, sans commentaire. En revanche, les entretiens ont fourni des informations complémentaires sur des pages de profil privées créées par les défunts de leur vivant, pages qui ne font cependant pas partie du corpus. Dans cette catégorie de pages, le producteur de la page est identique à l'objet de la page du vivant de l'usager puisqu'il s'agit d'une présentation numérique de soi par soi-même. Après le décès, ces pages peuvent continuer d'être alimentées, comme on l'a vu précédemment, par les proches et le système informatique. On a fait l'hypothèse que ce phénomène pouvait d'ailleurs expliquer en soi la sensation de récalcitrance identitaire ressentie par les proches. Or les entretiens ont montré des cas spécifiques, qui semblent ressurgir dans le corpus: le "ligateur autonyme" peut apparaittre actif encore postérieurement à la mort de l'usager, suscitant une situation d'énonciation incongrue dans laquelle le défunt («ligateur autonyme ») annonce lui-même sa mort à la troisième personne : « les funérailles auront lieu...». Comme rien ne l'indique techniquement, hormis l'usage de la troisième personne dans le contenu du post, le sujet effectif de l'énonciation n'est bien sûr pas le défunt, mais un proche ou un groupe de proches qui utilisent l'identifiant de ce dernier pour annoncer ses funérailles. Pour que cela soit possible Facebook ne donnant en aucun cas accès aux identifiants d'un tiers -, les proches en question doivent être parvenus d'une manière ou d'une autre à se procurer l'identifiant et le mot de passe du défunt après son décès. L'analyse appliquée de ces pages pourra être conduite dans des travaux ultérieurs pour interroger et expliciter les 
modalités énonciatives de passation de la parole du défunt à celle de ses proches et ses interprétations.

La présentation identitaire du créateur de la page mémoriale présente une ambiguïté technique dans les mémoriaux créés par les proches postérieurement au décès de l'usager, a fortiori pour les pages créées par les usagers de leur vivant et transformées en lieux d'hommage par les proches après leur décès. Toutefois, la mention du destinataire de l'hommage est clarifiée dans 14 pages sur 15 , par le recours à une formule ("Hommage à» et "Repose en paix»). Cette clarification porte sur la non-coïncidence entre le défunt et le créateur de la page mémoriale, mais n'est dans aucun cas du corpus explicite quant à la relation. L'analyse du sujet de l'énonciation dans le contenu des publications va permettre d'éclairer cet aspect.

\subsection{Le sujet textuel des publications et la signature}

L'identification du producteur de la page mémoriale n'est pas mis en évidence par le site Facebook, et le sujet de l'énonciation textuelle ne permet pas non plus toujours d'élucider rapidement ce mystère.

Dans le contenu des messages publiés sous le «ligateur autonyme» de la page, le «je» est utilisé dans 8 pages mémoriales. Sur 3 pages, «je » est utilisé comme pronom personnel principal, renvoyant à une fonction relationnelle bien identifiée, c'est-à-dire respectivement à une mère, à une sœur et à une fan du défunt. Dans les autres cas, il est employé en alternance avec des tournures à tendance impersonnelles (« nous » générique, «on», «il serait apprécié»). En particulier, lors de la création de la page, la tournure impersonnelle ou le «nous » générique est plutôt utilisé, et le sujet de l'énonciation se dévoile progressivement pour laisser place au « je » qui s'accompagne d'anecdotes ou de publications plus personnelles ou plus centrées sur le sujet, sa douleur et ses émotions. Par exemple «moi, ta petite maman », «dadou, ta protégée » peuvent apparaitre dans des posts publiés à quelques minutes d'intervalle, suggérant que les usagers se réunissent pour publier ensemble sur le profil du défunt.

L'usage du «nous» présente un flottement relativement à l'identification du sujet. En particulier dans les premiers posts du mémorial, le «nous» oscille entre un "nous» générique (ex. : «tu nous manques ») et un « nous » collectif référant à des personnes bien 
définies (ex. : «ton papa, tes sœurs et moi »), en passant par des « nous » implicitement définis (ex : la phrase « nous avons tous appris aujourd'hui » publiée sur la page d'hommage à un professeur réfère sans doute à une allocution présentée par son école en l'honneur du professeur défunt). L'énonciation de ce «nous», au sein des pages produites par les proches de manière intimiste, semble faire appel à des cadres interprétatifs relevant du cercle des proches. Un post évoque par exemple «notre lieu de recueillement dans ta chambre » complété par une photographie présentant des photographies encadrées au mur et décorées de guirlandes : ce post suggère que le « nous » réfère à la famille au sens étendu.

En somme, le « nous » semble référer implicitement à l'ensemble des proches intimes du défunt, susceptibles d'entretenir la page Facebook du défunt tout comme ils entretiennent sa sépulture. Certains utilisateurs prennent le soin d'apposer, dans le contenu d'une publication publiée sous l'identifiant générique, leur nom civil ou leur fonction dans la famille (ex. : sur le profil d'un bébé décédé : «ta marraine », " ta gnagnie / mamie »), ou encore leur relation au défunt (ex. sur le profil d'un jeune homme décédé : "ta protégée »). Ces appellations sont autant de signes appareillés à l'expression du deuil à la deuxième personne, celle de la mort de l'être cher, auquel les usagers s'adressent en considérant que le message pourra être compris par lui et s'adresse au défunt.

\subsection{Polémiques sur l'identité du créateur de la page}

Si le « nous » reste non explicité par les usagers (qui anime la page ?), certains créateurs des pages mémoriales rendent compte, sur le profil du défunt, de questions qui leur sont posées ainsi que de jugements portés sur leur légitimité à le faire lorsqu'ils ne sont pas de la famille, ou encore lorsqu'ils n'ont pas une position légitime dans la famille.

La créatrice d'une page mémoriale dédiée à une célébrité défunte s'exprime à ce sujet :

A tout ceux qui disent que j'ai bien fait de créer cette page, je voulais vous dire que je n'ai que 15 ans, mais j'était fan de cette actrice et mon film préféré était Comme t'y es belle, et elle me manque!! [sic]

Cette publication fait allusion d'une part aux jugements positifs portés selon le sujet à son projet, mais aussi au poids du jugement de 
F. GEORGES — Identité post mortem — 514

valeur référant à son statut social ("je n'ai que 15 ans mais») et au recours à une justification de son projet par son dévouement («j'étais fan », « elle me manque! »).

Dans le cas de l'hommage rendu à un couple de jeunes adultes, que ce soit du fait de leur appartenance à des familles différentes ou du fait du jugement négatif porté dans l'absolu sur cette personne, la créatrice de la page fait état de son irritation :

Je vais vous dire sincèrement, j'en est marre de recevoir ces messages qui disent « qui tiens cette page » qui ceci qui cela. Je suis la pour rendre Hommage a mes deux amis, parce que Ouais j'ai eu un mal chien a faire ce Deuil; a accepter cette tragédie. A admettre qu'ils n'étaient plus la ! J'ai pas envie de demander l'autorisation a qui que ce soit pour faire cette page et la faire vivre pour que mon deuil se fasse mieux... Ils méritent simplement cela; J'ai vécut mon enfance avec eux même si les 4 dernières année de Lycée nous on éloigner moi j'ai besoin de faire sa sa m'aide. Alors si cela ne vous plait pas; si vous n'accepter pas que je fasse cette page Personne ne vous oblige a rester la et a lire ce qui suit. Parce que moi je remarque que même les gens qui, de près ou de loin, les ont surement connu 1 voir 2 ans ont été touché par ce fléau. Merci simplement de respecter leurs dignités et l'hommage que je leur fait. Bonne nuit et Merci également a tout ceux qui la suive, et la font vivre chaque jour! [sic]

Comme la précédente, cette utilisatrice rend compte de jugements de valeur portés sur son projet mémorial, négatifs dans son cas, et justifie de même son entreprise par la douleur ressentie. La dernière expression est particulièrement signifiante relativement à la question de la «récalcitrance» en lien avec l'entretien de la page comme on entretiendrait le souvenir du défunt: ici, l'utilisatrice parle de «faire vivre» la page chaque jour. L'entretien de la page est bien conçu comme un entretien de la mémoire vivante du défunt.

Si l'énonciateur de la page mémoriale peut se dissimuler, il peut être l'objet de nombreuses incitations à mettre en lumière son identité, que ce soit sur la page ou plus fréquemment semble-t-il, par message privé. Il semble peser sur les épaules du créateur des pages une lourde charge symbolique, à travers les interrogations et justifications relatives à la question de la légitimité. Le rôle traditionnel d'entretien de la mémoire et de la sépulture par la famille proche semble en partie remis en question par les usages du numérique. En ligne, la

GEORGES, Fanny (2014) «Identité post mortem et nouvelles pratiques memoriales en ligne. L'identité du créateur de la page memoriale. », Les Cahiers du Gerse, Presses de l'Université du Québec. 
— Cahiers du gerse

production d'un mémorial devient le fait de quelqu'un de l'entourage qui trouve du sens dans la production d'un mémorial dans Facebook, et pour cette raison, cette personne ne coïncide pas toujours avec celle qui est jugée la plus légitime par l'entourage et la famille en particulier.

Dans les entretiens exploratoires que nous avons conduits auprès de jeunes adultes au sujet de mémoriaux en ligne rendant hommage à des amis proches ou plus éloignés, la question de la remise en question de la légitimité n'a pas été abordée spontanément par les usagers. Ces derniers rendaient compte néanmoins du fait que les mémoriaux sont généralement entretenus par le cercle amical le plus proche, sans que cette question n'ait été mentionnée comme problématique.

\section{Conclusion: l'énonciateur des pages mémoriales dans Facebook}

L'analyse de la présentation identitaire et du positionnement énonciatif des créateurs des 15 pages mémoriales à l'étude a montré que Facebook présente la particularité de rendre floues les limites entre le créateur de la page mémoriale (membre de l'entourage ou du cercle de fans) et l'objet de la page mémoriale (le défunt).

L'échantillon analysé montre que les usagers emploient des stratégies pour expliciter cette différence, notamment en mentionnant explicitement un hommage au défunt dans le titre de la page. Cette présentation complexe de l'énonciateur et son dévoilement progressif est propre à Facebook, et peut-être au domaine des réseaux socionumériques, qui ne sont pas initialement dédiés à la production de mémoriaux.

Pour ce qui est de l'identification individuelle des créateurs de pages mémoriales, plusieurs phases ont été observées et pourraient être réinterrogées dans un travail ultérieur. Une première phase correspond à l'annonce de la mort et privilégie les tournures impersonnelles. Assez rapidement (deux à trois jours suivant l'annonce de la mort), une seconde phase expose le créateur: il se présente à la première personne du singulier et présente son objet, dans notre corpus, en réaction à des sollicitations extérieures. Enfin, la troisième phase consiste en l'entretien régulier de la page, 
F. GEORGES — Identité post mortem — 516

privilégiant le passage au «je » exprimant le vécu du deuil et la vie avec la pensée du deuil.

Un second usage bien représenté dans le corpus est celui de la mention entre parenthèse, à la suite de l'identifiant de l'usager, du prénom du défunt et de la mention « Repose en paix ». Ces secondes formes de présentation du deuil, moins centrées sur le défunt, mettent l'accent sur la posture sociale du deuil.

\section{Bibliographie}

BELL, Genevieve. 2006. No more SMS from Jesus: Ubicomp, religion and techno-spiritual practices. UbiComp 2006: Ubiquitous Computing, 4206(2006), 141-158.

BLANDO, John A., GRAVES-FERRICK, Katie et GOECKE, Jo. 2004. Relationship differences in AIDS memorials. Omega, 49(2004), $27-42$.

BONENFANT, Maude (2008). «L'espace d'appropriation dans les jeux vidéo » dans Médiamorphoses, Paris, Institut National de l'Audiovisuel / Armand Colin, numéro 22, Février, pages 63 à 68.

BRUBAKER, Jed R. et HAYES, Gillian R. 2011. "We will never forget you [online]": An Empirical Investigation of Post mortem MySpace. Paper presented at the Computer Supported Cooperative Work (CSCW) conference 2011, Hangzhou (China), March 19-23, 2011. Available at: http://www.star-uci.org/wp-content/uploads/2011/01/deathpr464-brubaker.pdf [accessed June 2, 2014]

BRUBAKER, Jed R. et VERTESI, Janet. 2010. Death and the Social Network. Paper presented at the 28th Conference on Human Factors in Computing Systems (CHI 2010), Atlanta (GA, USA), April $10-15$

2010.

Available

at:

GEORGES, Fanny (2014) «Identité post mortem et nouvelles pratiques memoriales en ligne. L'identité du créateur de la page memoriale. », Les Cahiers du Gerse, Presses de l'Université du Québec. 
http://www.dgp.toronto.edu/ mikem/hcieol/subs/brubaker.pdf [accessed June 2, 2014].

COUTANT, Alexandre et STENGER, Thomas. 2010. Processus identitaire et ordre de l'interaction sur les réseaux socionumériques. Les Enjeux de l'Information et de la Communication, 1(2010). Available at: http://lesenjeux.u-grenoble3.fr/2010/Coutant-Stenger/Coutant-

Stenger.pdf [accessed May 30, 2014].

DE VRIES, Brian, et RUTHERFORD, Judy. 2004. Memorializing loved ones on the World Wide Web. Omega, 49(2004), 5-26.

ERTZSCHEID, Olivier, GALLEZOT, Gabriel et SIMONNOT, Brigitte. 2013. À la recherche de la mémoire du web: sédiments, traces et temporalités des documents en ligne. In Christine Barats (ed.), Manuel d'analyse du web, 53-68. Paris: Armand Colin.

FOONG, Pin Sym et KERA, Denisa. 2008. Applying reflective design to digital memorials. Paper presented at the International Workshop on Social Interaction and Mundane Technologies 2008 (SIMTech'08), Cambridge (UK), November 20-21, 2008. Available at: http://mundanetechnologies.com/goingson/workshop/cambridge/papers/FoongKera.pdf [accessed June 2, 2014].

GEORGES, Fanny. 2009. Identité numérique et Représentation de soi: analyse sémiotique et quantitative de l'emprise culturelle du web 2.0. Réseaux, 154(2009), 165-193.

GEORGES, Fanny. 2010. Identités virtuelles. Paris: Questions théoriques. $216 \mathrm{p}$.

GEORGES, Fanny et JULLIARD, Virginie. 2014. Identités numériques post mortem et usages mémoriaux du web à l'aune du genre. In Imad Saleh et Nasreddine Brouhai (eds.), Frontières numériques, 3550. Paris: L'Harmattan.

GEORGES, Fanny (2014) «Identité post mortem et nouvelles pratiques memoriales en ligne. L’identité du créateur de la page memoriale. », Les Cahiers du Gerse, Presses de l’Université du Québec. 
HALL, Christopher W. et REID, Robyn A. 2009. Adolescent bereavement over the deaths of celebrities. In David E. Balk et Charles A. Corr (eds.), Adolescent encounters with death, bereavement, and coping, 237-252. New York: Springer.

Haverinen, Anna Emilia. 2010. Memoria Virtualis - Digitalization of Mourning Rituals in Virtual Environments. Paper presented at the Nordic Network of Thanatology conference 2010, Aalborg (Denmark), November 24-26, 2010.

JANKÉLÉVITCH, Vladimir. 1977. La mort. Paris: Flammarion.

MERZEAU, Louise. 2009. Données post mortem. Hermès, 53(2009), 30.

MEUNIER, Jean-Pierre et PERAYA, Daniel. 2004. Introduction aux théories de la communication. Bruxelles: De Boeck.

ODOM, William, HARPER, Richard, SELLEN, Abigail, KIRK, David et BANKS, Richard. 2010. Passing on \& putting to rest: understanding bereavement in the context of interactive technologies. Paper presented at the Computer Human Interaction (CHI) conference 2010, Atlanta (Georgia, USA), April 10-15, 2010. Available at: http://dl.acm.org/citation.cfm?doid=1753326.1753601. [Accessed June 2, 2014].

PENE, Sophie. 2011. Facebook mort ou vif. Deuils intimes et causes communes. Questions de communication, 19(2011), 91-112.

RABATEL, Alain et FLORÉA, Marie-Laure. 2011. RePresentations of Death in the Information Media. Questions de communication, 19(2011), 7-28.

SOFKA, Carla J. 2009. Adolescents, technology, and the internet: coping with loss in the digital world. In David E. Balk et Charles A. Corr

GEORGES, Fanny (2014) «Identité post mortem et nouvelles pratiques memoriales en ligne. L'identité du créateur de la page memoriale. », Les Cahiers du Gerse, Presses de l’Université du Québec. 
— Cahiers du gerse

(eds.), Adolescent encounters with death, bereavement, and coping, 237252. New York: Springer.

WALTER, Joseph B., VAN DER HEIDE, Brandon, KIM, SangYeon, WESTERMAN, David et TOM TONG, Stephanie. 2008. The role of friends' appearance and behavior on evaluations of individuals on Facebook: Are we known by the company we keep? Human Communication research, 34(2008), 28-49.

WALTER, Tony, HOURIZI, Rachid, MONCUR, Wendy et PITSILLIDES, Stacey. 2012. Does the internet change how we die and mourn? An overview. Omega: Journal of Death et Dying, 64(4), 275302.

WRONA, Adeline. 2011. La vie des morts : jesuismort.com, entre bibliographie et nécrologie. Questions de communication, 19(2011), 7390. 